\title{
AN UNUSUAL TYPE OF EXPANSION PROBLEM*
}

\author{
BY
}

M. H. STONE

In the majority of papers on the expansion problems associated with linear differential systems or with integral equations, the chief aim is a proof that a function, arbitrary within the limits of certain restrictions as light as possible, can be expanded in a uniformly convergent series of functions arising from the differential system or the integral equation. As exceptions to the rule we may cite a number of papers dealing with differential systems for which the boundary conditions are of irregular type.t In the following paragraphs we present a study of the linear differential system of the first order, without the customary limitation that a certain coefficient in the differential equation remain positive; the results obtained are in sharp contrast not only with those found for the usual expansion problems and those already mentioned in connection with irregular boundary conditions, but also with the facts for the precisely analogous second order differential system. + A second point of some interest is the appearance of a normal orthogonal set of trigonometric functions differing from the normalized Fourier set but as closely related to it as are the sine and cosine sets. It is interesting that this exceedingly simple differential system seems to have escaped attention entirely. So far as we know, the existence of this gap in the theory of boundary value and expansion problems was first pointed out by Professor Birkhoff in a recent course of lectures on the theory of linear differential equations.

I. The FORMAL EXPANSION PROB! EM

We shall study here the two linear differential systems

$$
\begin{array}{lll}
u^{\prime}+\lambda p u=0, & 0 \leqq x \leqq 1 ; & u(0)-u(1)=0 ; \\
u^{\prime}+\lambda p u=0, & 0 \leqq x \leqq 1 ; & u(0)+u(1)=0 ;
\end{array}
$$

* Presented to the Society, march 1, 1924.

† Jackson, Proceedings of the American Academy of Arts and Sciences, vol.51 (1915-16), pp. 383-417; Hopkins, these Transactions, vol. 20 (1919), pp. 245-259.

$\ddagger$ Mason, these Transactions, vol. 8 (1907), pp. 427-432; Lichtenstein, Rendicorti del Circolo Matematico di Palermo, vol. 38 (1914), pp. 113-166. 
in which $\lambda$ is a complex parameter and $p$ is a bounded summable function with Lebesgue integral $\int_{0}^{1} p d x$ equal to unity, which satisfies the further requirement that a finite set of non-overlapping intervals $\varrho_{1}, \varrho_{2}, \cdots, \varrho_{n}$ can be determined such that

(a) the set covers the unit interval just once;

(b) on $\varrho_{i}$ either $p>0$ almost everywhere (i. e., except upon a set of points of zero measure) or $p<0$ almost everywhere;

(c) if $p>0$ on $\varrho_{i}$ almost everywhere, then $p<0$ almost everywhere on the two adjacent $\varrho$-intervals and vice versa.

It has been customary to assume $p \geqq l>0$ on the unit interval in discussions of expansion problems; in the present paper we dispense with this requirement. It will be apparent in the course of the development that there is no essential generalization in supposing $p$ of the character already described rather than continuous with a finite number of changes of sign. At this point we may also remark that the more general differential system

$$
\begin{aligned}
u^{\prime}+(\lambda p+q) u & =0, \quad a \leqq x \leqq b, \\
A u(a)+B u(b) & =0,
\end{aligned}
$$

where $\lambda$ and $p$ are restricted as before except that $\int_{0}^{1} p d x$ is merely different from zero, $q$ is any summable function, and $A$ and $B$ are real numbers different from zero, can be reduced by a series of real linear transformations of the independent variable and of the parameter together with a real transformation of the independent variable in the form

$$
u=u_{1} A\left(x_{1}\right), \quad A\left(x_{1}\right) \neq 0, \quad 0 \leqq x_{1} \leqq 1,
$$

to either (1) or (2) according as $A B<0$ or $A B>0$; the transformation can be effected in the sense that the new differential equation is true except possibly on a set of zero measure. If complex transformations are admitted (1) can be taken into (2), and conversely, in an analogous manner.

The adjoint differential systems for (1) and (2) are readily computed as

$$
\begin{array}{ll}
-v^{\prime}+\lambda p v=0, & -v(0)+v(1)=0 ; \\
-v^{\prime}+\lambda p v=0, & -v(0)-v(1)=0 .
\end{array}
$$

The differential equation $u^{\prime}+\lambda p u=0$ has the function

$$
u=e^{-\lambda P(x)}, \quad P(x)=\int_{0}^{x} p d x,
$$


as a solution in the sense that $u$ satisfies the differential equation except possibly on a set of measure zero. That this is the case may easily be verified by substitution, if it is remembered that $P^{\prime}(x)=p(x)$ almost everywhere, in accord with the theory of Lebesgue integration.* Furthermore, any other solution $u_{1}$ which is a Lebesgue integral is a constant multiple of $u$. To prove this we notice that

$$
\frac{d}{d x}\left(\frac{u_{1}}{u}\right)=e^{\lambda P(x)}\left[u_{1}^{\prime}+\lambda p u_{1}\right]=0 .
$$

On integration we find that $u_{1} / u=a$ constant. In the same way, the adjoint differential equation $-v^{\prime}+\lambda p v=0$ has a solution $v=e^{\lambda P(x)}$, unique except for a constant factor. When these solutions $u$ and $v$ are substituted in the boundary conditions of the two differential systems and in the adjoint boundary conditions, the characteristic equations

$$
\begin{aligned}
e^{\lambda} & =1, \\
e^{\lambda} & =-1
\end{aligned}
$$

are obtained. The roots of these equations, the characteristic numbers, are immediately seen to be

$$
\begin{array}{ll}
\lambda_{k}=2 k \pi i, & k=0, \pm 1, \pm 2, \cdots ; \\
\lambda_{k}=\pi i+2 k \pi i, & k=0, \pm 1, \pm 2, \cdots .
\end{array}
$$

In case we had admitted the equality $\int_{0}^{1} p d x=0$ we should have found here that every $\lambda$ is a characteristic value in (1), and that no $\lambda$ is a characteristic value in (2). Under our hypotheses the characteristic values form the discrete sets of numbers just determined. For solutions of (1) and (3.1) corresponding to distinct characteristic numbers $\lambda_{j}, \lambda_{k}$, we have, from the differential equations,

$$
\left(\lambda_{j}-\lambda_{k}\right) \int_{0}^{1} p u_{k} v_{j} d x=\int_{0}^{1}\left(u_{k} v_{j}^{\prime}+u_{k}^{\prime} v_{j}\right) d x=u_{k}(1) v_{j}(1)-u_{k}(0) v_{j}(0) .
$$

* Lebesgue, Leçons sur l'Intégration, Paris, 1904, pp. 124-125; de la Vallée Poussin, Intégrales de Lebesgue, Paris, 1916, §67. 
On account of the boundary conditions for (1) and (3.1) the last expression vanishes, so that

$$
\int_{0}^{1} p u_{k} v_{j} d x=0
$$

On the other hand, for solurions corresponding to the same characteristic number we are led to

$$
\int_{0}^{1} p u_{k} v_{k} d x=\int_{0}^{1} p e^{-\lambda_{k} P(x)} e^{+\lambda_{k} P(x)} d x=\int_{0}^{1} p d x=1 .
$$

In similar fashion we find for (2) and (3.2)

$$
\int_{0}^{1} p u_{k} v_{j} d x=\left\{\begin{array}{ll}
0 & k \neq j \\
1 & k=j
\end{array}\right\} .
$$

By analogy with Fourier series the following formal expansions of an arbitrary function integrable in the sense of Lebesgue are suggested.

$$
\begin{array}{ll}
\sum_{-\infty}^{+\infty} a_{k} u_{k}, & a_{k}=\int_{0}^{1} p f v_{k} d x \\
\sum_{-\infty}^{+\infty} a_{k} u_{k}, & a_{k}=\int_{0}^{1} p f v_{k} d x \\
\sum_{-\infty}^{+\infty} b_{k} v_{k}, & b_{k}=\int_{0}^{1} p f u_{k} d x \\
\sum_{-\infty}^{+\infty} b_{k} v_{k}, & b_{k}=\int_{0}^{1} p f u_{k} d x
\end{array}
$$

If we perform the formal operation of collecting the conjugate complex terms in these series, we find two related formal series of real terms with real coefficients. For the series (6.1) and (7.1) the terms with subscripts $+k$ and $-k$ respectively are conjugate complex; and the grouping of these terms yields one real series

$$
\begin{aligned}
& A_{0}+\sum_{1}^{\infty}\left\{A_{k} \sqrt{2} \cos 2 k \pi P(x)+B_{k} \sqrt{2} \sin 2 k \pi P(x)\right\} \\
& A_{0}=\int_{0}^{1} p f d x, \quad A_{k}=\int_{0}^{1} p f \sqrt{2} \cos 2 k \pi P(x) d x \\
& B_{k}=\int_{0}^{1} p f \sqrt{2} \sin 2 k \pi P(x) d x
\end{aligned}
$$


Similarly, the pairs of conjugate complex terms in the series (6.2) and (7.2) are those with subscripts $k \geqq 0,-k-1$ respectively; and the series obtainea by collecting terms is

$$
\begin{gathered}
\sum_{0}^{\infty}\left\{A_{k} \sqrt{2} \cos (2 k+1) \pi P(x)+B_{k} \sqrt{2} \sin (2 k+1) \pi P(x)\right\} \\
A_{k}=\int_{0}^{1} p f \sqrt{2} \cos (2 k+1) \pi P(x) d x \\
B_{k}=\int_{0}^{1} p f \sqrt{2} \sin (2 k+1) \pi P(x) d x
\end{gathered}
$$

It should be noted that the set of functions

$$
1, \quad V \overline{2} \cos 2 \pi x, \quad \sqrt{2} \sin 2 \pi x, \quad \sqrt{2} \cos 4 \pi x, \quad \sqrt{2} \sin 4 \pi x, \cdots,
$$

which serves as a basis for the formation of the series (8.1), is merely the ordinary Fourier set transformed to the unit interval and normalized.

$$
\text { II. THE CASE } p \equiv 1
$$

Before proceeding to more general considerations we propose to discuss the series (8.1) and (8.2) when $p \equiv 1$ almost everywhere. In this case $P(x) \equiv x$ so that the expansions appear as series formed from the two normal orthogonal sets

(9) $1, \quad \sqrt{2} \cos 2 \pi x, \quad \sqrt{2} \sin 2 \pi x, \quad \sqrt{2} \cos 4 \pi x, \quad \sqrt{2} \sin 4 \pi x, \ldots$,

$$
\sqrt{2} \cos \pi x, \quad \sqrt{2} \sin \pi x \quad \sqrt{2} \cos 3 \pi x, \quad \sqrt{2} \sin 3 \pi x, \ldots,
$$

on the interval $(0,1)$. Concerning the expansions of an arbitrary function integrable in the sense of Lebesgue we shall prove the

THEOREM I. The formal expansions of an arbitrary summable function in terms of the sets (9) and (10) on the interval $(0,1)$ are the formal expansions in terms of the normalized Fourier set for the interval $(0,2)$ of two functions $f_{1}$ and $f_{2}$ respectively.

Proof. The normalized Fourier set for the interval $(0,2)$ is seen to be (11) $\frac{1}{\sqrt{2}}, \cos \pi x, \sin \pi x, \cos 2 \pi x, \sin 2 \pi x, \cos 3 \pi x, \sin 4 \pi x, \cdots$

If we denote by $a_{k}, b_{k}$ the Fourier coefficients of a summable function in terms of (11) we have 


$$
\begin{aligned}
a_{0}=\frac{1}{\sqrt{2}} \int_{0}^{2} f d x & =\frac{1}{\sqrt{2}} \int_{0}^{1}\{f(x)+f(x+1)\} d x, \\
a_{2 k}=\int_{0}^{2} f \cos 2 k \pi x d x & =\int_{0}^{1}\{f(x)+f(x+1)\} \cos 2 k \pi x d x, \\
b_{2 k}=\int_{0}^{2} f \sin 2 k \pi x d x & =\int_{0}^{1}\{f(x)+f(x+1)\} \sin 2 k \pi x d x, \\
a_{2 k+1}=\int_{0}^{2} f \cos (2 k+1) \pi x d x & =\int_{0}^{1}\{f(x)-f(x+1)\} \cos (2 k+1) \pi x d x, \\
b_{2 k+1}=\int_{0}^{2} f \sin (2 k+1) \pi x d x & =\int_{0}^{1}\{f(x)-f(x+1)\} \sin (2 k+1) \pi x d x .
\end{aligned}
$$

If there is given an arbitrary summable function $f(x), 0 \leqq x \leqq 1$, we define $f_{1}(x)$ for the interval $(0,2)$ by the identities

$$
\begin{aligned}
f_{1}(x) & \equiv f(x), & & 0 \leqq x \leqq 1 \\
f_{1}(x+1) & \equiv f(x), & & 0<x \leqq 1 .
\end{aligned}
$$

Then the Fourier series of $f_{1}$ on $(0,2)$ has the coefficients

$$
\begin{gathered}
a_{0}=\sqrt{2} \int_{0}^{1} f d x, a_{2 k}=2 \int_{0}^{1} f \cos 2 k \pi x d x, \quad b_{2 k}=2 \int_{0}^{1} f \sin 2 k \pi x d x \\
a_{2 k+1}=b_{2 k+1}=0 .
\end{gathered}
$$

Furthermore the Fourier series thus set up,

$$
\frac{a_{0}}{\sqrt{2}}+\sum_{k=1}^{\infty}\left\{a_{2 k} \cos 2 k \pi x+b_{2 k} \sin 2 k \pi x\right\}
$$

is term for term the formal series for $f(x)$ in terms of $(9)$ on $(0,1)$.

Similarly, if we take $f_{2}(x)$ such that

$$
\begin{aligned}
f_{2}(x) & \equiv f(x), & 0 \leqq x \leqq 1 \\
f_{\mathbf{2}}(x+1) & \equiv-f(x), & 0<x \leqq 1
\end{aligned}
$$

there results

$$
\begin{gathered}
a_{0}=a_{2 k}=b_{2 k}=0 \\
a_{2 k+1}=2 \int_{0}^{1} f \cos (2 k+1) \pi x d x, \quad b_{2 k+1}=2 \int_{0} f \sin (2 k+1) \pi x d x
\end{gathered}
$$


and the formal series for $f_{2}$ becomes

$$
\sum_{k=0}^{\infty}\left\{a_{2 k+1} \cos (2 k+1) \pi x+b_{2 k+1} \sin (2 k+1) \pi x\right\}
$$

which is identified at once as the formal expansion of $f(x)$ in terms of (10) on $(0,1)$.

COROLlaRY I. The term-by-term difference of the formal series for $f(x)$ in terms of the Fourier set (9) and of the set (10) converges uniformly to zero on any closed interval interior to $(0,1)$.

Proof. This term-by-term difference is the Fourier series on $(0,2)$ for $f_{1}-f_{2} ;$ since $f_{1}-f_{2} \equiv 0,0 \leqq x \leqq 1$, it follows that this Fourier series converges uniformly to zero on any closed interval interior to $(0,1)$, by one of the most elementary properties of Fourier series.

COROLLARY II. If $f(x)$ is continuous and of bounded variation in a neighborhood on the right of $x=0$ and is continuous and of bounded variation in a neighborhood on the left of $x=1$, the formal series for $f(x)$ in terms of (10) converges at $x=0$ to $\frac{1}{2}(f(0+)-f(1-))$ and at $x=1$ to $\frac{1}{2}(-f(0+)+f(1-))$.

Proof. This is a direct consequence of the fact that the series is a Fourier series for $f_{2}(x)$ on $(0,2)$. It is sufficient to recall that under the conditions stated this Fourier series converges at $x=0$ to $\frac{1}{2}\left(f_{2}(0+)+f_{2}(2-)\right)$ and at $x=1$ to $\frac{1}{2}\left(f_{2}(1-)+f_{2}(1+)\right)$.

It is clear that a large number of properties of Fourier series will be carried over by Theorem I and Corollary I to the series formed from (10).* In particular there exists no summable function other than a function identically zero almost everywhere on $(0,1)$ such that its Fourier-like coefficients with respect to (10) all vanish. Again we see by Corollary I that all properties of divergence and of both uniform and non-uniform convergence and summability are shared alike on any closed interval interior to $(0,1)$ by the expansions formed from the Fourier set (9) and the set (10).

${ }^{*}$ It is of interest to notice that analogous considerations for the Fourier series on $(0,2 \pi)$ will lead to the series in terms of the sine set and of the cosine set on $(0, \pi)$,

$$
\sqrt{\frac{2}{\pi}} \sin x, \sqrt{\frac{\overline{2}}{\pi}} \sin 2 x, \cdots ; \quad \sqrt{\frac{1}{\pi}}, \sqrt{\frac{2}{\pi}} \cos x, \sqrt{\frac{2}{\pi}} \cos 2 x, \cdots .
$$

The term-by-term difference of the formal expansions of any summable function on $(0, \pi)$ in terms of these two sets converges uniformly to zero on any closed interval interior to $(0, \pi)$. For functions summable with summable square this was proved by Walsh and Wiener, Journal of Mathematics and Physics of the Massachusetts Institute of Technology, vol. 1 (1922), pp. 103-122, especially pp. 115-116. 
Gibbs' phenomenon is also common to the two series in the neighborhood of any interior point of the unit interval for which it occurs for the series from (9). The behavior of an expansion in terms of (10) on the entire unit interval may be discussed by means of the Fourier series for $f_{2}$ on $(0,2)$. The set $(10)$ is thus of considerable interest as a set of trigonometric functions distinct from the Fourier set but resembling it very closely and arising from a similar differential system. We may also observe that expansions in terms of (10) can be investigated separately from the Fourier series by means of an integral analogous to the Dirichlet integral which is central in the theory of the latter.

\section{The transformation $t=P(x)$}

It is evident at a glance that the transformation $t=P(x)$ is of fundamental importance in the discussion of the series (8.1) and (8.2) since, no matter what summable function is used in calculating the coefficients, any sum attributed to the series must be of the form $f[P(x)]$. The relation of this sum function, if it exists, to the function for which the formal series is written down will depend on the nature of this transformation and its application to the integrals which are the coefficients of the series. We therefore devote a section to a detailed study of the relation $t=P(x)$.

From the assumptions made in $\S I$ concerning the function $p(x)$ we may immediately deduce the following theorem which describes the manner of oscillation of the continuous function $P(x)=\int_{0}^{x} p d x$ :

Theorem II. The interval $(0,1)$ can be divided into a finite set of nonoverlapping intervals $\xi_{1}, \xi_{2}, \ldots, \xi_{m}$ such that

(a) the set covers the whole unit interval;

(b) on $\xi_{i}$ the function $P(x)$ either always increases or always decreases and furthermore $P(x)$ lies between consecutive integers: $k \leqq P(x) \leqq k+1$, $k=-M,-M+1, \cdots,-1,0,1, \cdots,+M$

(c) if on $\xi_{i}$ the function $P(x)$ always increases (decreases) and lies between consecutive integers $k$ and $k+1$, then on the two adjacent $\xi$-intervals $P(x)$ either always decreases (increases) or does not lie between the integers $k$ and $k+1$.

Proof. On any one of the intervals $\varrho_{i}$ of $\S \mathrm{I}, p(x)$ is either positive or negative almost everywhere. For the purpose of the argument we may assume the first possibility to be the case. Then for any two points $x_{1}$, $x_{2}$ on that interval we have $P\left(x_{2}\right)-P\left(x_{1}\right)=\int_{x_{1}}^{x_{2}} p d x>0, x_{2}>x_{1}$, since there exists on $\left(x_{1}, x_{2}\right)$ a measurable set of points with measure greater than zero on which $p(x)$ is positive. The same type of reasoning applies when $p(x)$ is negative on $\varrho_{i}$ rather than positive. 
Each interval $\varrho_{i}$, on which $P(x)$ is now known to be either always increasing or always decreasing, can be divided into a finite number of subintervals on each of which $P(x)$ lies between consecutive integers and on no two of which $P(x)$ lies between the same pair of integers. The number of sub-intervals thus determined is finite since $P(x)$ is continuous.

The sub-intervals just defined form a set of intervals $\xi_{1}, \xi_{2}, \ldots, \xi_{m}$ with all the properties set forth in the statement of the theorem.

It is now an exceedingly simple matter to determine the manner in which the intervals $\xi_{i}$ are transformed by the relation $t=P(x)$. We have

THEOREM III. The transformation $t=P(x)$ takes the set of intervals $\xi_{i}$ into a set of intervals $\tau_{i}$ such that

(a) $\tau_{i}$ has positive or negative sense according as $P(x)$ increases or decreases on $\xi_{i}$

(b) the algebraic sum of the intervals $\tau_{i}$ is the unit $t$-interval;

(c) $\boldsymbol{\tau}_{i}$ lies between successive integers.

Proof. The interval $\xi_{i}:\left(a_{i}, b_{i}\right)$ clearly goes over into the interval $\tau_{i}$ : $\left[P\left(a_{i}\right), P\left(b_{i}\right)\right]$. Statements $(a)$ and $(c)$ are then made apparent by Theorem II $(b)$. It is also easy to see that if $0, x_{1}, x_{2}, \cdots, x_{n-1}, 1$ are the end points of the intervals $\varrho_{i}$ of $\S I$ the algebraic sum of the intervals $\tau_{i}$ is found by adding the intervals $\left[P(0), P\left(x_{1}\right)\right],\left[P\left(x_{1}\right), P\left(x_{2}\right)\right], \cdots$, $\left[P\left(x_{n-1}\right), P(1)\right]$ algebraically. The sum is then seen to be the interval $[P(0), P(1)]$ which is precisely the interval $(0,1)$.

Before going on to discuss the transformation of integrals, we shall state two necessary lemmas of a general nature. Of these we shall prove the

Lемма I. If $t=t(x)$ is the Lebesgue integral $\int_{a}^{x} g(x) d x+a^{\prime}$ of $a$ summable function $g(x)$ which is positive almost everywhere $a \leqq x \leqq b$, then

(a) $t=t(x)$ has a unique inverse $x=x(t)$ which is a one-valued always increasing function of $t, a^{\prime} \leqq t \leqq b^{\prime}$;

(b) $x(t)$ is equal to the Lebesgue integral $\int_{a^{\prime}}^{t} \frac{d t}{g[x(t)]}+a$.

If $t=t(x)$ is the Lebesgue integral $\int_{a}^{x} g(x) d x+a^{\prime}$ of a summable function $g(x)$ which is negative almost everywhere $a \leqq x \leqq b$, then

(a) $t=t(x)$ has a unique inverse $x=x(t)$ which is a one-valued always decreasing function of $t, b^{\prime} \leqq t \leqq a^{\prime}$

(b) $x(t)$ is equal to the Lebesgue integral $\int_{a^{\prime}}^{t} \frac{d t}{g[x(t)]}+a$.

Proof. As we have already seen in the proof of Theorem $\Pi, t(x)$ is an always increasing continuous function of $x$ when $g(x)$ is positive almost everywhere on $(a, b)$; statement $(a)$ of the first part of the lemma follows 
immediately from this fact. Turning now to $(b)$ in the first part of the lemma, we examine the equality

$$
\frac{x(t+h)-x(t)}{h}=\frac{h^{\prime}}{t\left(x+h^{\prime}\right)-t(x)},
$$

where $h, h^{\prime}$ vanish together. The reciprocal of the term on the right has the limit $g(x)$ almost everywhere; since $g(x)$ is positive almost everywhere the term itself has a finite limit almost everywhere. Corresponding to the set of zero measure on the $x$-axis for which the limit does not exist or is not equal to $g(x)$ there is a set of zero measure on the $t$-axis. To establish this statement we note that the measure of the set of points on the $t$-axis corresponding to a finite or denumerably infinite set of nonoverlapping intervals $E$ on the $x$-axis is equal to the integral $\int_{E} g d x$. We can now enclose any set of zero measure on the $x$-axis in an open point set of measure less than $\delta *$, that is, in a finite or denumerably infinite set of non-overlapping closed intervals $E+$. By choosing $\delta$ sufficiently small we can make the measure of $E^{\prime}$, which corresponds to $E$ and therefore contains the set corresponding to the set of zero measure on the $x$-axis, less than any preassigned positive $\varepsilon$ :

$$
m\left(E^{\prime}\right)=\int_{E} g(x) d x<\varepsilon, \quad m(E)<\delta .
$$

Thus we must have the result that $\lim _{h \rightarrow 0}(x(t+h)-x(t)) / h$ exists and is equal to $1 / g[x(t)]$ almost everywhere, $a^{\prime} \leqq t \leqq b^{\prime}$.

If we can show that $x(t)$ is a Lebesgue integral we can then state that the function is the indefinite integral of its differential coefficient, $x=\int_{a^{\prime}}^{t} \frac{d t}{g[x(t)]}+a$. It is well known that a necessary and sufficient condition that a function be an integral in the sense of Lebesgue is that it be absolutely continuous. + We shall prove that this is true for $x(t)$; that is, we shall show that $\sum\left|x\left(b_{i}^{\prime}\right)-x\left(a_{i}^{\prime}\right)\right|$ formed for any set of nonoverlapping closed intervals $E^{\prime}$ can be made uniformly small by taking $m\left(E^{\prime}\right)$ sufficiently small. In other words, if we are given any positive $\varepsilon$, however small, we can find a positive $\delta$ such that if $m\left(E^{\prime}\right)<\delta$ then $m(E)<\varepsilon$, where $E$ is the set of intervals on the $x$-axis corresponding to $E^{\prime}$. For the set of points on which $g \leqq \sqrt{\delta}$ is of measure $\eta(\delta)$ vanishing with $\delta$; we pick $V \bar{\delta}+\eta(\delta)<\bar{\varepsilon}$ and require $m\left(E^{\prime}\right)<\delta$.

* de la Vallée Poussin, loc. cit., § 21.

† de la Vallée Poussin, loc. cit., $§ 14$.

$\ddagger$ Vitali, Atti della Reale Accademia di Torino, vol. 40 (1905), pp. 1021-1034; Lebesgue, loc. cit., p. 129, footnote. 
Splitting $E$ into the measurable sets of points $E_{1}, E_{2}$ on which $g>\sqrt{\delta}$, $g \leqq \sqrt{\delta}$ respectively, we see that

whence

$$
\sqrt{\delta} m\left(E_{1}\right) \leqq \int_{E_{1}} g d x \leqq \int_{E} g d x=m\left(E^{\prime}\right)<\delta,
$$

$$
m(E)=m\left(E_{1}\right)+m\left(E_{2}\right) \leqq \sqrt{\delta}+\eta(\delta)<\varepsilon .
$$

The proof of the first part of the lemma is thus completed. The second part, in which $g(x)$ is negative almost everywhere, is proved in the same manner.

The second lemma we state without proof. It is

LEмма II.* If $x=x(t)$ is the Lebesgue integral of a summable function $h(t)$ which is positive (negative) almost everywhere on $\left(a^{\prime}, b^{\prime}\right)$, then for any summable function $f(x)$

$$
\begin{gathered}
\int_{a}^{b} f(x) d x=\int_{a^{\prime}}^{b^{\prime}} f[x(t)] \frac{d x(t)}{d t} d t=\int_{a^{\prime}}^{b^{\prime}} f[x(t)] h(t) d t \\
b=x\left(b^{\prime}\right), \quad a=x\left(a^{\prime}\right) .
\end{gathered}
$$

With the aid of Lemmas I and II we are now able to demonstrate Theorem IV. If $p(x) f(x)$ is summable, $0 \leqq x \leqq 1$, then

$$
\int_{\xi_{i}} p f d x=\int_{\tau_{i}} p\left[X_{i}(t)\right] f\left[X_{i}(t)\right] \frac{d X_{i}(t)}{d t} d t=\int_{\tau_{i}} f\left[X_{i}(t)\right] d t
$$

where $X_{i}(t)$ is the inverse of $t=P(x)$ for the interval $\xi_{i}$; and, conversely, if $f(t)$ is summable and is defined outside the unit interval by the periodic relation $f(t+1)=f(t)$ or by the anti-periodic relation $f(t+1)=-f(t)$, then

$$
\int_{\tau_{i}} f(t) d t=\int_{\xi_{i}} f[P(x)] \frac{d P(x)}{d x} d x=\int_{\xi_{i}} p(x) f[P(x)] d x .
$$

Proof. On the interval $\xi_{i}$, the function $t=P(x)$ is the Lebesgue integral of a function positive (negative) almost everywhere on that interval, so that by Lemma I we can invert $t=P(x)$ by means of the function

$$
X_{i}=\int_{a_{i}^{\prime}}^{t} \frac{d t}{p\left[X_{i}(t)\right]}+a_{i}
$$

${ }^{*}$ Lebesgue, Annales de la Faculté des Sciences de Toulouse, ser. 3, vol. 1 (1909), pp. 25-117, especially p. 44; Hobson, The Theory of Functions of a Real Variable, second edition, Cambridge University Press, 1921, § 440, pp. 592-595. 
for $t$ on $\tau_{i}:\left(a_{i}^{\prime}, b_{i}^{\prime}\right)$ and $x$ on $\xi_{i}:\left(a_{i}, b_{i}\right)$. We then apply the second Lemma to each of the two integrals $\int_{\xi_{i}} p f d x, \int_{\tau_{i}} f d t$ remembering that $1 / p\left[X_{i}(t)\right]$ is positive almost everywhere or negative almost everywhere on $\boldsymbol{\tau}_{i}$ according as $p$ is positive or negative almost everywhere on $\xi_{i}$. It should be noted that since $p$ is bounded, $p f$ is certainly summable if $f$ is.

In order to pass to the consideration of integrals like those which appear as the coefficients in (8.1) and (8.2), we introduce new concepts. If we define $X_{i}(t)$ by the periodic relation $X_{i}(t+1)=X_{i}(t)$ for all the intervals $\tau_{i}+k, k=0, \pm 1, \ldots$ then for some $k$, say $k_{i}$, one of these intervals will lie entirely on the unit interval and thus

$$
\int_{\tau_{i}} f\left[X_{i}(t)\right] d t=\int_{\tau_{i}+k_{i}} f\left[X_{i}(t)\right] d t
$$

The new interval $\tau_{i}+k_{i}$ will still have definite positive or negative sense; to eliminate this circumstance, we let $\boldsymbol{\tau}_{i}^{\prime}$ be the interval $\boldsymbol{\tau}_{i}+k_{i}$ taken in the positive sense. We can then write

$$
\int_{\tau_{i}} f\left[X_{i}(t)\right] d t=\int_{\tau_{i}^{\prime}} \operatorname{sgn} \tau_{i} f\left[X_{i}(t)\right] d t
$$

where $\operatorname{sgn} \boldsymbol{\tau}_{i}=+1$ if $\boldsymbol{\tau}_{i}$ has positive sense and $\operatorname{sgn} \boldsymbol{\tau}_{i}=-1$ if $\boldsymbol{\tau}_{i}$ has negative sense. With a view to the discussion of the series (8.1) we now define a function

$$
g_{1}(t)=f_{11}(t)+f_{12}(t)+\cdots+f_{1 m}(t),
$$

where $f_{1 i} \equiv \operatorname{sgn} \tau_{i} f\left[X_{i}\right]$ on $\tau_{i}^{\prime}$ and is identically zero elsewhere on $(0,1)$. Similarly, with a view to the treatment of (8.2) we define a second function

$$
g_{2}(t)=f_{21}(t)+f_{22}(t)+\cdots+f_{2 m}(t),
$$

where $f_{2 i} \equiv(-)^{k_{i}} \operatorname{sgn} \tau_{i} f\left[X_{i}\right]$ on $\boldsymbol{\tau}_{i}^{\prime}$ and is identically zero elsewhere on $(0,1)$. These two functions are so related to $f(x)$ that we shall call them the first and second $P$-average functions for $f(x)$, respectively. The introduction of these functions is prompted by considerations which will be more apparent later in our work.

We now prove two theorems on integrals which will serve as the basis for our analysis of the series (8.1) and (8.2). We begin with 
Theorem V. If $p(x) f(x)$ is summable, then $g_{1}(t)$ and $g_{2}(t)$ are summable and

$$
\int_{0}^{1} p(x) f(x) d x=\int_{0}^{1} g_{1}(t) d t .
$$

If $p(x) f^{2}(x)$ is also summable, then $g_{1}(t)$ and $g_{2}(t)$ are both summable with summable square. If $f(x)$ is bounded, then $g_{1}(t)$ and $g_{2}(t)$ are also bounded.

Proof. If $p(x) f(x)$ is summable, then, by Theorem IV:

and

$$
\int_{\xi_{i}} p f d x=\int_{0}^{1} f_{1 i}(t) d t
$$

$$
\int_{\xi_{i}} p f d x=(-1)^{k_{i}} \int_{0}^{1} f_{2 i} d t
$$

so that $f_{1 i}$ and $f_{2 i}$ are summable. Since $g_{1}(t)$ is a linear combination of the $f_{1 i}$ and $g_{2}(t)$ of the $f_{2 i}$, both $g_{1}(t)$ and $g_{2}(t)$ are summable. The addition of the equalities

gives us the fact that

$$
\int_{\xi_{i}} p f d x=\int_{0}^{1} f_{1 i} d t
$$

$$
\int_{0}^{1} p f d x=\int_{0}^{1} g_{1}(t) d t .
$$

If $p f^{2}$ is summable also, then $\int_{\xi_{1}} p f^{2} d x$ exists and by Theorem IV and the definition of the functions $f_{1 i}, f_{2 i}$ is equal to

$$
\int_{0}^{1} f_{1 i}^{2} d t=\int_{0}^{1} f_{2 i}^{2} d t
$$

Thus $f_{1 i}^{2}$ and $f_{2 i}^{2}$ are summable so that this is also true of $g_{1}$ and $g_{2}$. We may note that if $\int_{0}^{1} p f^{2} d x$ exists the inequality

$$
\begin{aligned}
\left|\int_{0}^{1} p f d x\right| & \leqq \int_{0}^{1}|p f| d x \\
& =\int_{0}^{1} \sqrt{|p|} \sqrt{|p|}|f| d x \leqq \sqrt{\int_{0}^{1}|p| d x \cdot \int_{0}^{1}|p| f^{2} d x}
\end{aligned}
$$

proves the existence of $\int_{0}^{1} p f d x$. 
Lastly, if $f$ is bounded so are $f_{1 i}$ and $f_{2 i}$. In consequence $g_{1}$ and $g_{2}$ are also bounded.

The other theorem serves as a sort of converse to the one we have just proved. Its statement follows:

Theorem VI. If $h(t)$ is summable and is defined outside the unit interval by the periodic relation $h(t+1)=h(t)$ or the anti-periodic relation $h(t+1)=-h(t)$, then $p(x) h[P(x)]$ is summable and

$$
\int_{0}^{1} p h[P] d x=\int_{0}^{1} h(t) d t
$$

If $h(t)$ is in addition of summable square, then $p h^{2}[P]$ is summable. If $h(t)$ is bounded, then so is $h[P(x)]$.

Proof. In any of the three cases we can write

$$
\int_{0}^{1} h(t) d t=\sum_{1}^{m} \int_{\tau_{i}} h(t) d t=\sum_{1}^{m} \int_{\xi_{i}} p(x) h[P(x)] d x=\int_{0}^{1} p(x) h[P(x)] d x .
$$

Thus $p h[P]$ is summable. In case we have $h^{2}(t)$ summable we may replace $h(t)$ by $h^{2}(t)$ in the equalities just written down, obtaining $\int_{0}^{1} h^{2}(t) d t=\int_{0}^{1} p h^{2}[P] d x$ and in this way proving that $p h^{2}[P]$ is summable. It is obvious that if $h(t)$ is bounded, $h[P]$ is also bounded.

With Theorems V and VI we are prepared to treat the two series (8.1) and (8.2). It seems in place, however, to say a word about the possibility of extending the results of this section to transformations associated with a somewhat more general class of functions $p$. To obtain analogues of Theorems II and III it is sufficient to require that it be possible to determine a denumerably infinite set of non-overlapping intervals $\varrho_{1}, \varrho_{2}, \varrho_{3}, \ldots$ covering the unit interval except for a set $X$ of zero measure and such that on $\varrho_{i}$ the function $p(x)$ is either positive or negative almost everywhere. The analysis necessary to establish a theorem parallel to Theorem III is somewhat more complicated than that which we have found sufficient for the type of function $p(x)$ we are considering. Theorem IV remains the same. By investigating some simple convergence questions we can set up the $P$-average functions for any function $f(x)$, but Theorems V and VI are no longer true, at least for the general class of functions $p(x)$ just defined. To bring out the essential points of the treatment which we have outlined, we shall take up a simple example.

We shall define $p(x)$ so as to be constant on each of a denumerably infinite set of intervals $\varrho_{1}, \varrho_{2}, \ldots$. It is well known that the infinite series 
$1+2 \sum_{1}^{\infty}\left(1 / n^{2}\right)$ converges to a positive constant $C$. The interval $\varrho_{1}$ shall be of length $1 / C$ and shall have $x=1$ as an end point. Then $\varrho_{2}$ shall abut on $\varrho_{1}$ and be of length $1 / 4 C, \varrho_{3}$ shall abut on $\varrho_{2}$ and be of length $1 / 4 C$. In general, $\varrho_{i+1}$ shall abut on $\varrho_{i}$, and $\varrho_{2 k}, \varrho_{2 k+1}$ shall each be of length $1 /\left((k+1)^{8} C\right)$. Then $\sum_{1}^{\infty} m\left(\varrho_{i}\right)=1$. We shall denote the end points of the intervals $\varrho_{1}, \varrho_{2}, \ldots$ by $1=a_{0}>a_{1}>a_{2} \ldots$. Now we put

$$
\left.\begin{array}{rl}
p(x) & \equiv+C, \quad a_{2 k+1}>x>a_{2 k+2} \\
p(x) & \equiv-C, \quad a_{2 k}>x>a_{2 k+1} \\
p\left(a_{k}\right) & \equiv 0
\end{array}\right\} \quad k=0,1,2, \cdots
$$

A few simple computations show that $P(x)=\int_{0}^{x} p(x) d x$ is a linear function of $x$ on each $\varrho$-interval, while $P\left(a_{2 k}\right)=1 /(k+1)^{2}, k=0,1,2, \cdots$ and $P\left(a_{2 k+1}\right)=0, k=0,1,2, \ldots$. The $\varrho$-intervals of this example are precisely the $\xi$-intervals analogous to those of Theorem II. By the transformation $t=P(x)$ there corresponds to the interval $\varrho_{2 k+1}$ the interval $\tau_{2 k+1}:\left(0,1 /(k+1)^{2}\right)$ taken in the positive sense, and to the interval $\varrho_{2 k}$ the interval $\tau_{2 k}:\left(0,1 /(k+1)^{2}\right)$ taken in the negative sense. Since all the intervals $\boldsymbol{\tau}_{i}$ are on the unit interval, the two $P$-average functions coincide in this case. In constructing this one we will obtain instead of a finite sum an infinite series which, however, has the peculiarity that on any interval $(\varepsilon, 1), \varepsilon>0$, it is actually a finite sum. As an example we may take the bounded summable function which is identical to 1 on $\varrho_{1}, \varrho_{3}, \varrho_{5}, \ldots$, and identical to zero elsewhere. We have at once

$$
\int_{\rho_{2 k+1}} p d x=\int_{\tau_{2 k+1}} d t
$$

Thus if we put $f_{2 k+1}(t) \equiv 1$ on $\tau_{2 k+1}$ and let it vanish identically elsewhere we find that the $P$-average functions for this particular $f(x)$ are given by the infinite series $g(t)=\sum_{k=0}^{\infty} f_{2 k+1}(t)$. It is quickly seen that $g(t) \equiv k$ on $\left[1 /(k+1)^{2}, 1 / k^{2}\right]$. Now $f(x)$, being bounded, is of summable square; but, on the other hand, we see that

$$
\int_{0}^{1} g^{2} d t=\sum_{k=1}^{\infty} k^{2}\left[\frac{1}{k^{2}}-\frac{1}{(k+1)^{2}}\right]=\sum_{k=1}^{\infty} \frac{2 k+1}{(k+1)^{2}},
$$

a divergent series. Thus $g(t)$ is not of summable square, and Theorem $\mathrm{V}$ is not true as a whole for this transformation. In the same way we can 
show that, although $\frac{1}{2} t^{-\frac{1}{2}}$ is summable, $\frac{1}{2} p(x) P^{-\frac{1}{2}}(x)$ is not; for if $\frac{1}{2} p P^{-\frac{1}{2}}$ were summable its integral over the set of intervals $\varrho_{1}, \varrho_{8}, \varrho_{5}, \ldots$ would exist and would be given by $\sum_{k=0}^{\infty} \int_{\rho_{2 k+1}} p P^{-\frac{1}{2}} d x$. This last expression turns out to be a divergent series since

$$
\sum_{k=0}^{\infty} \int_{\rho_{\mathbf{2}+1}} p P^{-\frac{1}{2}} d x=\sum_{k=0}^{\infty} \int_{\tau_{\mathbf{2}+1}} \frac{1}{2} t^{-\frac{1}{2}} d t=\sum_{k=0}^{\infty}\left[t^{\frac{1}{2}}\right]_{t=0}^{t=\frac{1}{(k+1)^{2}}}=\sum_{k=0}^{\infty} \frac{1}{k+1} .
$$

Thus Theorem VI is not true as a whole for this special case.

The example which we have just discussed makes it clear why we have not attempted to take $p(x)$ of more general character, and serves to indicate along what lines any generalization can be expected to take place. With this we may leave the developments of this section.

\section{The BEHAVIOR OF THE FORMAL EXPANSIONS}

Instead of discussing the series (8.1) and (8.2) alone we shall consider a wider class of series including these two as special cases. If we are given any normal orthogonal set $\varphi_{1 k}(t)$, where $\varphi_{1 k}(t+1)=\varphi_{1 k}(t)$, we can apply Theorem VI to the summable function $\varphi_{1 i}(t) \varphi_{1 k}(t)$, whence

$$
\int_{0}^{1} \varphi_{1 i} \varphi_{1 k} d t=\int_{0}^{1} p \varphi_{1 i}[P] \varphi_{1 k}[P] d x=\left\{\begin{array}{ll}
0 & i \neq k \\
1 & i=k
\end{array}\right\} .
$$

The set (9) is a special case. Similarly, if we are given a set of normal orthogonal functions $\varphi_{2 k}(t)$ where $\varphi_{2 k}(t+1)=-\varphi_{2 k}(t)$ we find

$$
\int_{0}^{1} p \varphi_{2 i}[P] \varphi_{2 k}[P] d x=\left\{\begin{array}{ll}
0 & i \neq k \\
1 & i=k
\end{array}\right\} .
$$

The set (10) is a special example. It is natural to form from the sets

$$
\begin{array}{llll}
\varphi_{11}[P], & \varphi_{12}[P], & \varphi_{13}[P], & \cdots \\
\varphi_{21}[P], & \varphi_{22}[P], & \varphi_{23}[P], & \cdots
\end{array}
$$

formal series $\sum_{1}^{\infty} a_{1 k} \varphi_{1 k}[P]$ and $\sum_{1}^{\infty} a_{2 k} \varphi_{2 k}[P]$ respectively for any function of the class for which $a_{1 k}=\int_{0}^{1} p f \varphi_{1 k}[P] d x$ and $a_{2 k}=\int_{0}^{1} p f \varphi_{2 k}[P] d x$ 
exist. If $\varphi_{1 k}, \varphi_{2 k}$ are bounded then this class of functions is at least the class of all functions for which $p(x) f(x)$ is summable. If $\varphi_{1 k}, \varphi_{2 k}$ are summable with summable square then this class is at least the class of all functions for which $p(x) f^{2}(x)$ is summable. By Theorem VI the Lebesgue integrals $\int_{0}^{1} p \varphi_{1 k}^{2}[P] d x, \int_{0}^{1} p \varphi_{2 k}^{2}[P] d x$ exist. Because of the existence of $\int_{0}^{1} p f^{2} d x$ the inequalities

$$
\begin{aligned}
& \left|\int_{0}^{1} p f \varphi_{1 k}[P] d x\right| \leqq \int_{0}^{1}\left|p f \varphi_{1 k}[P]\right| d x \\
& =\int_{0}^{1} \sqrt{|p|}|f| V|p|\left|\varphi_{1 k}[P]\right| d x \leqq \sqrt{\int_{0}^{1}|p| f^{2} d x \cdot \int_{0}^{1}|p| \varphi_{1 k}^{2}[P] d x} \\
& \left|\int_{0}^{1} p f \varphi_{2 k}[P] d x\right| \leqq \int_{0}^{1}\left|p f \varphi_{2 k}[P]\right| d x \\
& =\int_{0}^{1} \sqrt{|p|}|f| V|p|\left|\varphi_{2 k}[P]\right| d x \leqq \sqrt{\int_{0}^{1}|p| f^{2} d x \cdot \int_{0}^{1}|p| \varphi_{2 k}^{2}[P] d x}
\end{aligned}
$$

demonstrate the summability of the measurable functions $p f \varphi_{1 k}, p f \varphi_{2 k}$.

We come next to

The0REM VII. The Fourier-like coefficients of $f(x)$ in terms of the set of finctions $\varphi_{11}[P], \varphi_{12}[P], \ldots$ are the Fourier-like coefficients of the first $P$-average function for $f(x)$ in terms of the set $\varphi_{11}(t), \varphi_{12}(t), \ldots$; and the Fourier-like coefficients of $f(x)$ in terms of the set $\varphi_{21}[P], \varphi_{22}[P], \cdots$ are the Fourier-like coefficients of the second P-average function for $f(x)$ in terms of the set $\varphi_{21}(t), \varphi_{22}(t), \ldots$.

Proof. If $G_{1 k}(t)$ is the first $P$-average function for the product $f \varphi_{1 k}[P]$ then, by Theorem V, $\int_{0}^{1} G_{1 k} d t=\int_{0}^{1} p f \varphi_{1 k}[P] d t$. Now $G_{1 k}(t)$ when evaluated is precisely $g_{1}(t) \varphi_{1 k}(t)$ where $g_{1}$ is the first $P$-average function for $f(x)$. Hence $a_{1 k}=\int_{0}^{1} p f \varphi_{1 k}[P] d x=\int_{0}^{1} g_{1}(t) \varphi_{1 k}(t) d t$. In the same way, if $G_{2 k}(t)$ is the first $P$-average function for the product $f \varphi_{2 k}[P]$ and $g_{2}$ is the second $P$-average function for $f(x)$ then

$$
G_{2 k}(t)=g_{2}(t) \varsigma_{2 k}(t), \quad a_{2 k}=\int_{0}^{1} p f \varphi_{2 k}[P] d x=\int_{0}^{1} g_{2}(t) \varphi_{2 k}(t) d t
$$

CoRollary I. If $\int_{0}^{1} p f^{2} d x$ exists, a series formed from $\sum_{0}^{\infty} a_{1 k} \varphi_{1 k}[P] b y$ grouping its terms suitably will converge essentially uniformly to $g_{1}^{*}[P]$ where 
$g_{1}^{*}(t+1)=g_{1}^{*}(t) ;$ and a series formed from $\sum_{1}^{\infty} a_{2 k} \varphi_{2 k}[P]$ by grouping its terms properly will converge essentially uniformly to $g_{2}^{*}[P]$ where. $g_{2}^{*}(t+1)=-g_{2}^{*}(t)$.

Proof. By the hypothesis that $\int_{0}^{1} p f^{2} d x$ exists we have the fact that the two $P$-average functions for $f(x)$ are summable with summable square. Consider the first of these, $g_{1}(t)$. By the Riesz-Fischer theorem, in the form given to it by Weyl, there exists a series formed by grouping the terms of the series $\sum_{1}^{\infty} a_{1 k} \varphi_{1 k}(t)$ which will converge essentially uniformly to a function $g_{1}^{*}(t)$, where $g_{1}(t)-g_{1}^{*}(t)$ is a function all of whose coefficients with respect to $\varphi_{11}(t), \varphi_{12}(t), \ldots$ vanish. $t$ By essentially uniform convergence we mean that, given any decreasing sequence of positive $\varepsilon^{\prime \prime}$ s with limit zero, $\varepsilon_{1}^{\prime}>\varepsilon_{2}^{\prime}>\varepsilon_{3}^{\prime}>\ldots$, we can find on the unit interval a sequence of measurable point sets $E_{1}^{\prime}, E_{2}^{\prime}, E_{3}^{\prime}, \ldots$ each contained in the succeeding set, with $m\left(E_{k}^{\prime}\right)>1-\varepsilon_{k}^{\prime}$, such that on each set of the sequence the series converges uniformly to its limit. On the unit $x$-interval we now have a sequence of point sets $E_{1}, E_{2}, E_{3}, \ldots$ where $E_{k}$ is the set of points for which $t=P(x)$ takes on values congruent (modulo 1) to a point of $E_{k}^{\prime}$. We see that on $E_{k}$ the series formed by grouping the terms of $\sum_{1}^{\infty} a_{1 k} \varphi_{1 k}[P]$ converges uniformly to $g_{1}^{*}[P]$ because of the periodicity of the functions $\varphi_{1 k}(t)$. Now $C E_{k}$, the set complementary to $E_{k}$, consists of all the points of the unit $x$-interval for which $t=P(x)$ is not congruent (modulo 1) to a point of $E_{k}^{\prime}$; that is, of all the points for which $t=P(x)$ is congruent to a point of $C E_{k}^{\prime}$. Thus $C E_{k}$ can be obtained as the sum of the point sets $e_{1 k}, e_{2 k}, e_{3 k}, \cdots, e_{m k}$, where $e_{i k}$ is the set on $\xi_{i}$ corresponding by the relation $t=P(x)$ to a set congruent (modulo 1) to $C E_{k}^{\prime}$. Since $m\left(C E_{k}^{\prime}\right)<\varepsilon_{k}^{\prime}$ we can enclose $C E_{k}^{\prime}$ in an open set $O_{k}^{\prime}$ of measure $<2 \varepsilon_{k}^{\prime}$. On the interval $\xi_{i}$ the set $o_{i k}$ corresponding to $O_{k}^{\prime}$ contains $e_{i k}$. Since for values of $t$ on $\boldsymbol{\tau}_{i}$ we have

$$
x=X_{i}(t)=\int_{\tau_{i}} \frac{d t}{p\left[X_{i}\right]}+a_{i}
$$

it follows that $m\left(o_{i k}\right)$ is given by the Lebesgue integral of $\operatorname{sgn} \boldsymbol{\tau}_{i} / p\left[X_{i}\right]$ taken over the points of $O_{k}^{\prime}$ on $\boldsymbol{\tau}_{i}^{\prime}$. From this point on we apply the arguments we used in discussing a similar situation in the proof of Lemma I. By choosing an $\varepsilon_{k}^{\prime}$ small enough we can make $m\left(C E_{k}\right)=\sum_{i=1}^{m} m\left(e_{i k}\right)$ $\leqq \sum_{i=1}^{m} m\left(o_{i k}\right)$ less than any preassigned positive $\varepsilon$. Thus, given a sequence of decreasing positive $\varepsilon_{k}$ 's with limit zero, we can find a subsequence of $E_{1}, E_{2}, E_{2}, \ldots$, say $E_{1}^{\prime \prime}, E_{2}^{\prime \prime}, E_{3}^{\prime \prime}, \ldots$, such that $m\left(E_{k}^{\prime \prime}\right)>1-\varepsilon_{k}$. The

† Plancherel, Rendiconti del Circolo Matematico di Palermo, vol. 30 (1910), pp. 289-335, chap. I. 
series formed by grouping the terms of $\sum_{1}^{\infty} a_{1 k} \varphi_{1 k}[P]$ therefore converges essentially uniformly to the function $g_{1}^{*}[P]$ where $g_{1}^{*}(t+1)=g_{1}^{*}(t)$. If the set $\varphi_{1 k}(t)$ is closed with respect to all functions summable with summable square-that is, if the only function of this class which has all zero coefficients with respect to the set is identically zero except on a set of measure zero-then $g_{1}^{*}(t)=g_{1}(t)$ almost everywhere. Exactly the same sort of reasoning applies to the function $g_{2}(t)$ and the series $\sum_{1}^{\infty} a_{2 k} \varphi_{2 k}(t)$, the only difference being that here the functions $\varphi_{2 k}(t)$ are anti-periodic instead of periodic.

COROLlaRY II. If the set $\varphi_{1 k}(t)$ is closed with respect to all functions summable with summable square, and if $f_{1}(x)$ and $f_{2}(x)$ are two functions such that $\int_{0}^{1} p f_{1}^{2} d x$ and $\int_{0}^{1} p f_{2}^{2} d x$ exist, then a necessary and sufficient condition that $f_{1}(x)$ and $f_{2}(x)$ have the same coefficients with respect to $\varphi_{11}[P], \varphi_{12}[P], \cdots$ is that their first P-average functions coincide almost everywhere. If the set $\varphi_{1 k}$ is closed with respect to all summable functions, and if $f_{1}(x)$ and $f_{2}(x)$ are two functions such that $\int_{0}^{1} p f_{1} d x$ and $\int_{0}^{1} p f_{2} d x$ exist, then a necessary and sufficient condition that $f_{1}(x)$ and $f_{2}(x)$ have the same coefficients with respect to the set $\varphi_{11}[P], \varphi_{12}[P], \ldots$ is that their first $P$-average functions coincide almost everywhere. Similar statements involving the second P-average functions can be made for the set $\varphi_{21}[P]$, $\varphi_{22}[P], \cdots$.

As we have arready remarked in $\S$ II the sets (9) and (10) are closed with respect to all summable functions.

Corollary I to Theorem VII suggests a theorem which we shall prove independently as

THEOREM VIII. If the function $P(x)$ is not monotone, there are infinitely many non-null functions whose P-average functions are both identically zero.

Proof. By a non-null function we mean any function which is not a null function; and a null function is any function identically zero except on a set of measure zero. When we assume that $P(x)$ is not monotone we assume that there are two or more of the intervals $\varrho_{i}$ described in $\S \mathrm{I}$. For the sake of definiteness we shall assume that on $\varrho_{1}:(0, a)$ the function $P(x)$ increases from zero to $P(a)$ while on $\varrho_{2}:(a, b)$ it decreases from $P(a)$ to $P(b)$. If $P(b) \geqq 0$ we consider the interval $R_{1}$ made up of $\varrho_{2}$ and the sub-interval of $\varrho_{1}$ for which $P(b) \leqq P(x) \leqq P(a)$; in case $P(b) \leqq 0$ we take the interval $R_{2}$ composed of $\varrho_{1}$ and the sub-interval of $\varrho_{2}$ for which $0 \leqq P(x) \leqq P(a)$. If there is given any bounded summable function $h(t)$ defined for all values of $t$ on the interval $0 \leqq t \leqq P(a)$, we set $f(x)$ identically equal to $h[P]$ on $R_{1}$ or $R_{2}$ as the case may be, and identically equal to zero elsewhere, $0 \leqq x \leqq 1$. It is then not difficult 
to see by reference to the definitions of the two $P$-average functions for $f(x)$ that both these functions vanish in the case of the bounded summable function which we have constructed. All the coefficients of $f(x)$ with respect to the sets $\varphi_{11}[P], \varphi_{12}[P], \ldots$ and $\varphi_{21}[P], \varphi_{22}[P], \ldots$ exist and vanish by Theorem VII. It is, of course, unnecessary but simpler to confine ourselves to bounded functions.

Theorem VII, Corollary I, and Theorem VIII reveal the striking peculiarities of the expansions of $\S I$ and their generalizations; peculiarities entirely new, so far as we are informed, in expansion problems. For any function $f(x)$ such that $\int_{0}^{1} p(x) f^{2}(x) d x$ exists, the formal series will always represent in a certain sense a definite function, which will be of the specialized form $g_{1}^{*}[P]$ or $g_{2}^{*}[P]$. It is clear that if $\int_{0}^{1} p f^{2} d x$ exists then $f(x)$ can be represented by its formal series in terms of a closed set $\varphi_{11}[P], \varphi_{12}[P], \cdots$ if and only if its functional values are so distributed that it is of the form $g_{1}[P], g_{1}(t+1)=g_{1}(t)$ except possibly on a set of measure zero; and by its formal series in terms of a closed set $\varphi_{21}[P], \varphi_{22}[P], \ldots$ if and only if its functional values are so distributed that it is of the form $g_{2}[P], g_{2}(t+1)=-g_{2}(t)$ except possibly on a set of measure zero. There are infinitely many functions all having the same formal expansions in terms of the two general sets of functions which we are discussing, and this quite apart from any considerations of the closure of the sets $\varphi_{11}(t), \varphi_{12}(t), \cdots$ and $\varphi_{21}(t), \varphi_{22}(t), \ldots$. The remarks which have just been made are, of course, expected to apply only in case $P(x)$ is not monotone. If $P(x)$ is monotone the expansions have no unusual properties.

The detailed consideration of a formal series here is thrown back on to that of the series $\sum_{1}^{\infty} a_{1 k} \varphi_{1 k}(t)$ for $g_{1}(t)$ or the series $\sum_{1}^{\infty} a_{2 k} \varphi_{2 k}(t)$ for $g_{2}(t)$. We do not intend to go deeply into any such discussion, but will close with one theorem bearing on the series (8.1) and (8.2):

THEOREM IX. If the function $f(x)$ is of bounded variation and if its first $P$-average function is extended by the periodic relation $g_{1}(t+1)=g_{1}(t)$ then the formal series (8.1) converges at every point $x$ for which $P(x)=t_{0}$ to the value $\frac{1}{2}\left(g_{1}\left(t_{0}+0\right)+g_{1}\left(t_{0}-0\right)\right)$; and if its second $P$-average function is extended by the relation $g_{2}(t+1)=-g_{2}(t)$ then the formal series (8.2) converges at every point $x$ for which $P(x)=t_{0}$ to the value $\frac{1}{2}\left(g_{2}\left(t_{0}+0\right)+g_{2}\left(t_{0}-0\right)\right)$.

Proof. The function $f(x)$ can be represented as the difference of two monotone increasing functions $f_{1}$ and $f_{2}$ because it is of bounded variation. Now the functions $f_{1 i}(t)$ and $f_{2 i}(t)$ used in defining the $P$-average functions for $f(x)$ are constant multiples of $f\left[X_{i}(t)\right]$ on $\boldsymbol{\tau}_{i}^{\prime}$ and zero elsewhere. $X_{i}(t)$ is periodic of period 1 and is monotone on $\boldsymbol{\tau}_{i}^{\prime}$. Since a monotone function 
of a monotone function is monotone, both $f_{1}\left[X_{i}(t)\right]$ and $f_{2}\left[X_{i}(t)\right]$ are monotone on $\boldsymbol{x}_{i}^{\prime}$; and $f\left[X_{i}\right]=f_{1}\left[X_{i}\right]-f_{\mathrm{a}}\left[X_{i}\right]$ is therefore of bounded variation on $\boldsymbol{\tau}_{i}^{\prime}$. Thus $g_{1}(t)$ and $g_{2}(t)$ are also of bounded variation, being linear combinations of functions of bounded variation. Now the series for $g_{1}$ in terms of the set (9) is the Fourier series for a function of bounded variation and therefore converges to $\frac{1}{2}\left(g_{1}\left(t_{0}+0\right)+g_{1}\left(t_{0}-0\right)\right)$ at $t_{0}$. The substitution of $P(x)$ for $t$ gives the first part of the theorem as stated. Likewise, as we saw in Theorem I, the series for $g_{2}(t)$, where $g_{8}(t+1)=-g_{2}(t)$, in terms of (10) is the Fourier series on the interval $(0,2)$ for a function of bounded variation. The series converges at the point $t_{0}$ to the value $\frac{1}{2}\left(g_{2}\left(t_{0}+0\right)+g_{2}\left(t_{0}-0\right)\right)$. The substitution of $P(x)$ for $t$ completes the theorem.

Other theorems of a similar nature will readily suggest themselves to the reader. We shall not go into any further detail, having obtained by the work up to this point a fairly complete idea of the character of the expansion problems arising from the two differential systems (1) and (2).

HARVARD UNIVERSITY,

Cambridge, Mass. 